REVIEW

\title{
Pulmonary tumour thrombotic microangiopathy
}

AQ2

Laura C. Price, Athol U. Wells, and Stephen J. Wort

\begin{abstract}
Purpose of review
Pulmonary tumour thrombotic microangiopathy (PTTM) describes tumour cell microemboli with occlusive fibrointimal remodelling in small pulmonary arteries, veins and lymphatics. Progressive vessel occlusion ultimately results in pulmonary hypertension, which is often severe and rapid in onset. PTTM is associated with carcinomas, notably gastric carcinoma, with vascular endothelial growth factor and platelet-derived growth factor signalling implicated in driving the intimal remodelling. PTTM is a rare cause of pulmonary hypertension, but given that up to a quarter of autopsy specimens from patients dying of carcinoma show evidence for PTTM, it is probably underdiagnosed.
\end{abstract}

AQ5

\section{Recent findings}

Until recently, prognosis in PTTM was universally abysmal from weeks to a few months. Diagnostic utilities AQ6 include aspiration of tumour cells at wedged right heart catheterization, HRCT findings and computed tomography-positron emission tomography (CT-PET), although definitive diagnosis requires histological analysis. Reports of PTTM treated with a combination of targeted pulmonary vasodilator therapies, anticoagulation, specific chemotherapy and platelet-derived growth factor inhibition, for example using imatinib, suggest that these approaches can prolong survival.

\section{Summary}

PTTM is increasingly recognized as an important cause of pulmonary hypertension, often in patients presenting with new-onset pulmonary hypertension and as yet undiagnosed malignancy. Prospects of survival are improving with targeted combination therapy, and early recognition and diagnosis are likely to be the key factors to improve outcome.

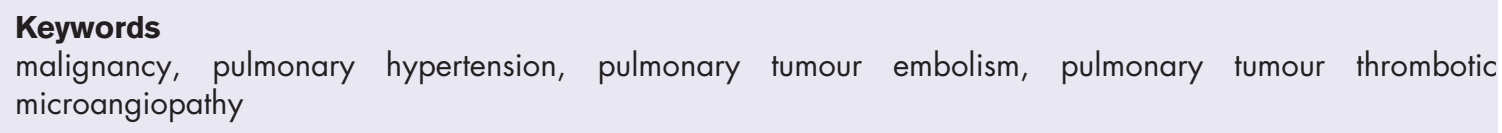

\section{INTRODUCTION}

Pulmonary tumour thrombotic microangiopathy (PTTM) is a rare pulmonary manifestation of malignancy, and was first described by von Herbay in 1990 [1]. Prior to the description of PTTM, the simple occlusion of small pulmonary arteries by coagulated tumour cells was termed pulmonary tumour embolism that was first described in 1930 in a patient who died following a hydatidiform mole [2]. In 1990, von Herbay analysed autopsy specimens from patients with carcinoma. He documented PTTM as a condition that appeared to progress from pulmonary tumour embolism, from microscopic (nonocclusive) tumour cell emboli to occlusive fibrocellular intimal proliferation, mainly within small precapillary pulmonary vessels. The resulting progressive increase in vascular resistance leads to pulmonary hypertension [1]. PTTM may present as pulmonary hypertension of unknown origin. It may be difficult to diagnose, and is difficult to distinguish from pulmonary tumour embolism except following histological examination of lung tissue. Until recently, PTTM was universally a postmortem finding, with an appalling prognosis $[3,4]$. Recent advances, however, suggest improvements in short-term outcomes.

We review the epidemiology, pathophysiology, clinical presentation, investigations and management of PTTM, including recent cases of PTTM in which ante-mortem diagnosis provide hope for this devastating condition.

\section{DEFINITION}

PTTM is characterized by the presence of nonocclusive pulmonary vascular tumour microembolic

Royal Brompton Hospital, London, UK

Correspondence to Dr Laura C. Price, Royal Brompton Hospital, London, AQ4 UK. E-mail: I.price08@imperial.ac.uk

Curr Opin Pulm Med 2016, 22:000-000

DOI:10.1097/MCP.0000000000000297 


\section{KEY POINTS}

- PTTM is a rare but probably underdiagnosed cause of pulmonary hypertension.

- PTTM is associated with carcinoma, usually gastric carcinoma.

- Characteristic features are tumour microemboli with occlusive fibrointimal proliferation in small pulmonary vessels.

- Early diagnosis and use of targeted chemotherapy are likely to improve outcome.

- Targeting growth factors including PDGF have marginally improved survival in recent reports, but formal trials are awaited.

'nests' with evidence for activation of coagulation, obliterative intimal proliferation and ultimately pulmonary hypertension $[5,6]$. Pulmonary tumour embolism is a related condition in which coagulated tumour cells obstruct the pulmonary microcirculation without changes in pulmonary vessel architecture [7]. By definition, pulmonary metastases and larger pulmonary emboli are excluded.

\section{INCIDENCE/EPIDEMIOLOGY}

When the primary diagnosis of malignancy is known, PTTM usually relates to a carcinoma, usually an adenocarcinoma [4,5]. Autopsy series of carcinomas suggest that the reported prevalence is $1-3 \%$ $[5,8]$. Most reported cases of PTTM have been described in Japan, which is likely to reflect the high prevalence of gastric carcinoma. PTTM occurs in $16-27 \%$ cases of gastric carcinoma $[4,5,9]$, especially the mucinous, signet ring and poorly differentiated subtypes $[3,5,9]$. PTTM is less commonly associated with breast [10], bladder [11], ovarian clear cell [12], hepatocellular [13] and gallbladder carcinoma [14]. A common feature is the presence of metastatic disease, often with lymphangitic spread $[5,12]$.

\section{PATHOGENESIS}

There appears to be an intimate association between nests of tumour cells and surrounding fibrointimal proliferation in pulmonary arteries and veins, which ultimately leads to vessel occlusion (Fig. 1a and b). The proliferating intimal cells are both endothelial and nonendothelial cell (i.e. myofibroblastic) in origin [5]. Cancer cell attachment is postulated to cause intimal (endothelial cell) damage, activation of coagulation and initiation of proliferation through growth factors and cytokine release/ expression, including tissue factor, vascular endothelial growth factor (VEGF) $[4,8,9]$ and plateletderived growth factor (PDGF) [9,15"]. PDGF is known to initiate macrophage recruitment and upregulate VEGF on cancer cells, which may further increase endothelial cell proliferation. Interestingly, PDGF inhibition (using imatinib, see later) has led to recanalization of previously occluded small pulmonary arteries in a case of PTTM related to gastric cancer [15"].

Vascular inflammation is also likely to contribute to the pathogenesis of PTTM. Dense perivascular and intimal accumulation of macrophages have been reported $\left[16,17^{\mathbf{*}}, 18\right]$ (and Fig. 1b). Putative mediators include osteopontin, a cytokine and adhesive protein that is implicated in tumoral thrombosis and neointima formation [17"] as well as promotion of progression and metastasis of cancer [19]. In PTTM, macrophages stain for osteopontin and CD44 (an osteopontin receptor) [17"], with tumour cells and proliferating fibrointimal cells also overexpressing both CD44 and osteopontin, as well as PDGF and VEGF [16,17"]. Therefore, crosstalk between tumour cells, macrophages and intimal cells may occur through the osteopontin-CD44 axis to drive both tumorigenesis and ongoing macrophage recruitment. Macrophage-derived factors, such as interleukin-6 (IL-6), as implicated in PAH [20], may also contribute to the fibrointimal proliferation in PTTM. Tumour cell nests are also evident in pulmonary veins and lymphatics $[9,10,18]$, with evidence for lymphatic tumour invasion (Fig. 1c). Fibrointimal proliferation in pulmonary veins may be analogous to the remodelling of pulmonary veins seen in patients with distal CTEPH [21]. Putative interactions between tumour cells, macrophages and vascular cells are summarized in Fig. 2.

It should also be emphasized that, although certain similarities exist, the pathogenesis of pulmonary vessel remodelling is unlike PAH in several respects: there are no studies that demonstrate plexiform lesions, PASMC or adventitial remodelling, or evidence for augmented ET-1 or reduced nitric oxide or prostacyclin signalling characteristic of PAH.

\section{Haematological aspects}

Carcinomas promote coagulation by several mechanisms [22], such as the release of microvesicles containing tissue factor [9], and through interaction (by mucinous carcinomas) with selectin on platelets [23]. Binding of tissue factor with factor VII and calcium ultimately leads to fibrin deposition and platelet activation to form clots. Progressive disseminated intravascular coagulation (DIC) consumes fibrin, with release of fibrinogen breakdown 


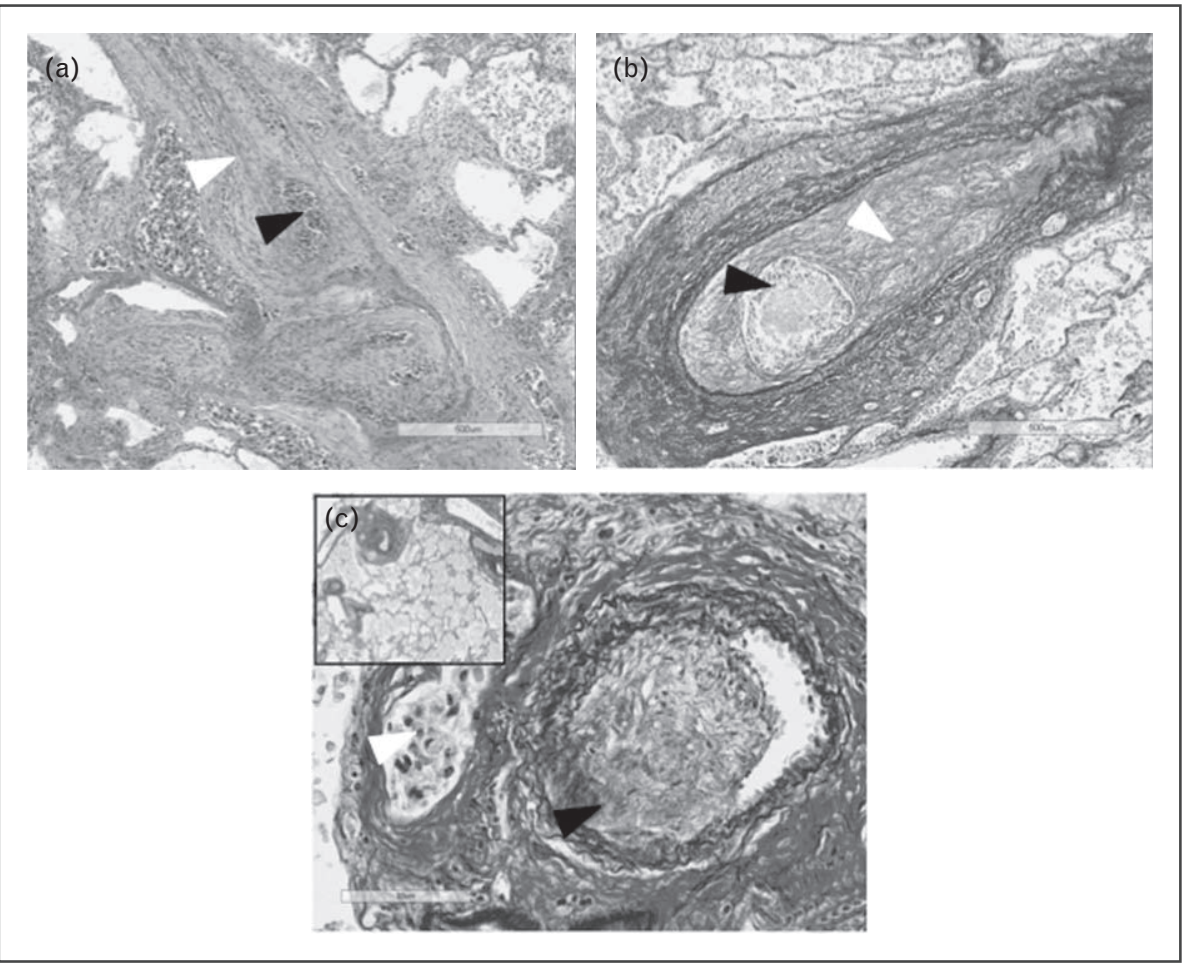

FIGURE 1. Lung histology from PTTM case related to severe pulmonary hypertension in a patient without a history of known malignancy. (a) Post-mortem section with occlusion of medium sized pulmonary arterial lumen by fibrointimal proliferation (of fibroblasts and collagen) (white arrow) and tumour emboli (black arrow) (H\&E, $\times 4$.3). (b) Post-mortem section showing a medium sized pulmonary artery with two elastic layers, with a normal sized smooth muscle layer. There is exaggerated luminal occlusion by fibrointimal thickening (white arrow) surrounding nests of tumour emboli (black arrow). The adventitia contains lymphatic tumoral thrombi. Increased alveolar macrophages are seen in surrounding lung (EVG stain, $\times 4.6)$.

(c) Evidence for fibrointimal proliferation within the lumen of small pulmonary veins (black arrow) and tumour involvement of accompanying lymphatics (white arrow). Inset: veins close to the centrilobular bronchovascular bundles show eccentric fibrointimal remodelling $(E V G$ stain, $\times 3.4)(E V G, \times 28.4)$. PTTM, pulmonary tumour thrombotic microangiopathy.

products (FDPs) or D dimers. Usually DIC is rapid and associated with bleeding, whereas in malignancy, 'compensated DIC' can occur, wherein the rate of consumption of coagulation factors and platelets is slow; thrombotic manifestations exceed bleeding events [24]. Evidence for raised D dimer or FDPs has been a uniform finding in PTTM $[8,24]$, with features of microangiopathic haemolytic anaemia (MAHA) or DIC in half of cases at presentation [8,24-26].

\section{Development of pulmonary hypertension}

Pulmonary hypertension develops in PTTM as a consequence of progressive vascular fibrointimal stenosis and occlusion [3,5]. Autopsy specimens from patients with pulmonary hypertension show variable luminal occlusion with a median of $30 \%$ of vessels occluded in symptomatic patients [6] and widespread severe luminal narrowing a feature in severe pulmonary hypertension [9].

\section{CLASSIFICATION OF PULMONARY TUMOUR THROMBOTIC MICROANGIOPATHY}

Pulmonary tumour embolism was first classified in 2004 within 'group 4' of the World Health Organization (WHO) classification system of pulmonary hypertension [27], and in the most recent update, 'other intrarscular tumours' remains within group 4.2.2 (ESC@5). PTTM, with increasing recognition and understanding of its multifaceted mechanisms, now lies within group 5 , that is reflecting multifactorial and/or unclear mechanisms of pulmonary hypertension with 'tumoral obstruction', being within the subgroup 5.4 (Galie, ESCQD).

\section{CLINICAL PRESENTATION}

Patients with PTTM usually present with rapidly progressive exertional breathlessness, hypoxia and progression right ventricle (RV) dysfunction [8]. Onset is usually 3 weeks to 6 months prior to 


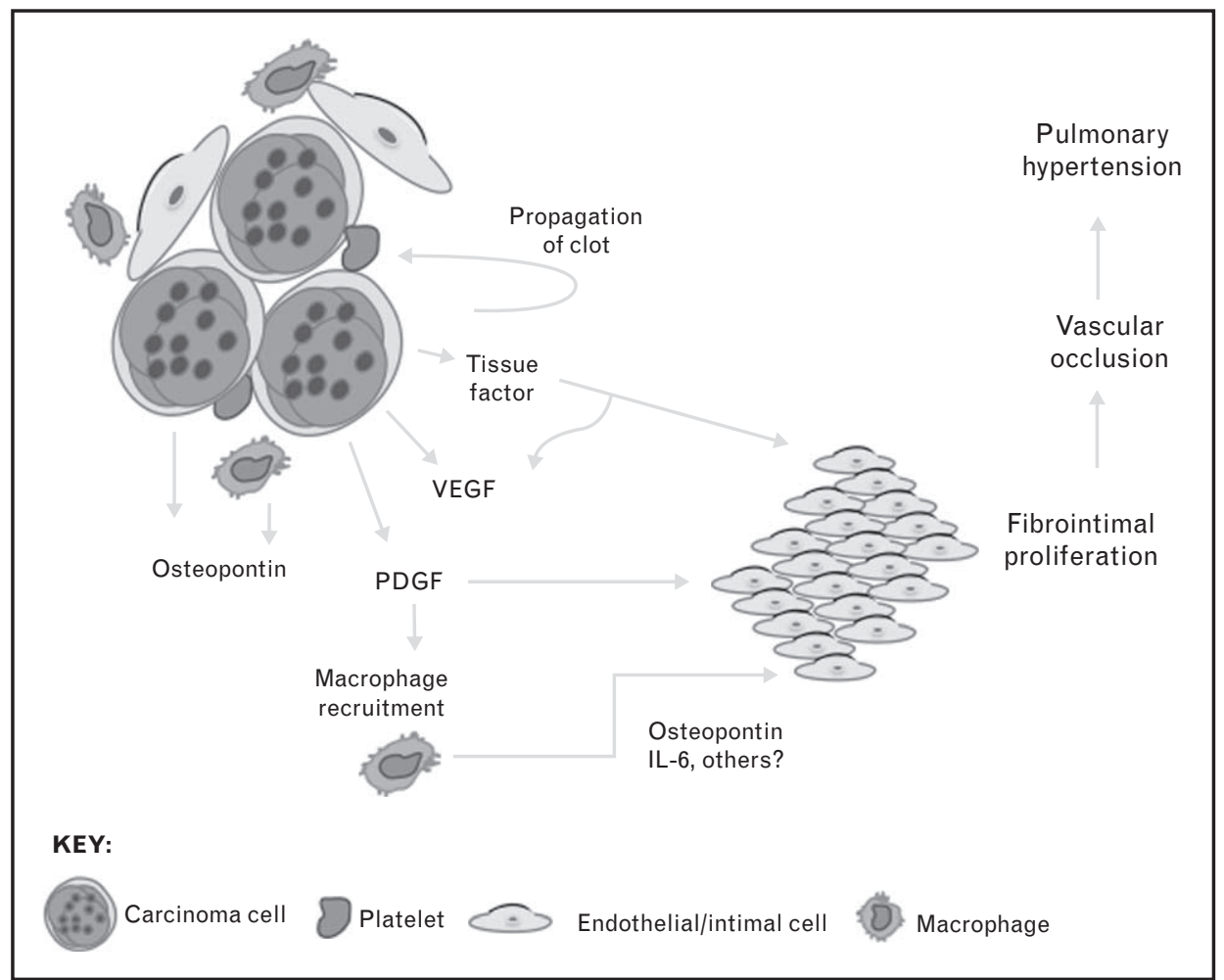

FIGURE 2. Proposed mechanisms for fibrointimal proliferation in PTTM. Small nests of carcinomatous cells lodge in pulmonary vessels, including small precapillary arteries (via haematogenous spread) and to veins and lymphatics (by lymphatic invasion). Tumour cell and endothelial cell interaction initiate clot formation, for example-through expression and release of tissue factor. Tumour cells release further cytokines including vascular endothelial growth factor (VEGF) and platelet-derived growth factor (PDGF) that initiate macrophage recruitment and intimal (endothelial cell and nonendothelial cell like, i.e. myofibroblastic) proliferation. Tissue factor also upregulates VEGF expression on tumour cells, which is angiogenic to intimal cells. PDGF-A and $B$ are expressed on tumour cells, and antiphospho-PDGFR-alpha on vascular endothelial cell and gastric carcinoma cell indicates PDGF signalling activation through autocrine and paracrine mechanisms. The cytokine and adhesive protein osteopontin is expressed on tumour cells in PTTM, within PTTM lesions and on the primary tumour, as well as on proliferating fibromuscular intimal cells and macrophages with the PTTM lesions, and is likely to be a key driver for intimal cell growth. Perivascular CD68-positive macrophages are noted and also reside within intimal layers. Macrophages also stain for CD44, the adhesion molecule, which interacts with OPN to induce chemotaxis of T cells and macrophages, propagation of local inflammation and intimal proliferation (through other known macrophage-derived proproliferative factors including IL-6). The resulting fibrointimal proliferation is present in both precapillary and postcapillary small pulmonary vessels, and has occlusive characteristics, hence the development of pulmonary hypertension and hypoxia in PTTM. Direct contact with tumour nests is not universal in all vessels where remodelling is present. IL-6, interleukin-6; PTTM, pulmonary tumour thrombotic microangiopathy.

presentation $[4,5,28]$. Cough may predate dyspnoea. Haemoptysis, chest and abdominal pain, which may reflect liver metastases or venous congestion, have also been reported [26]. The progression of symptoms correlates with HRCT findings prior to the onset of pulmonary hypertension, which may be a few weeks to months later [28]. Fatigue is also reported [29].

Depending on the timing of presentation, symptoms and signs of pulmonary hypertension may or not be present. These include a prominent pulmonary second heart sound, raised jugular venous pressure, and further signs of RV decompensation.
However, these signs may often be absent even with confirmed pulmonary hypertension [30].

\section{INVESTIGATIONS FOR PULMONARY TUMOUR THROMBOTIC MICROANGIOPATHY}

Diagnosis of PTTM can be challenging and many findings are nonspecific. ECG may show signs of RV strain in the presence of pulmonary hypertension. Chest radiography is often normal. Bloods demonstrate raised D-dimer or FDPs [8], and MAHA in some cases. Pulmonary function tests show pulmonary 


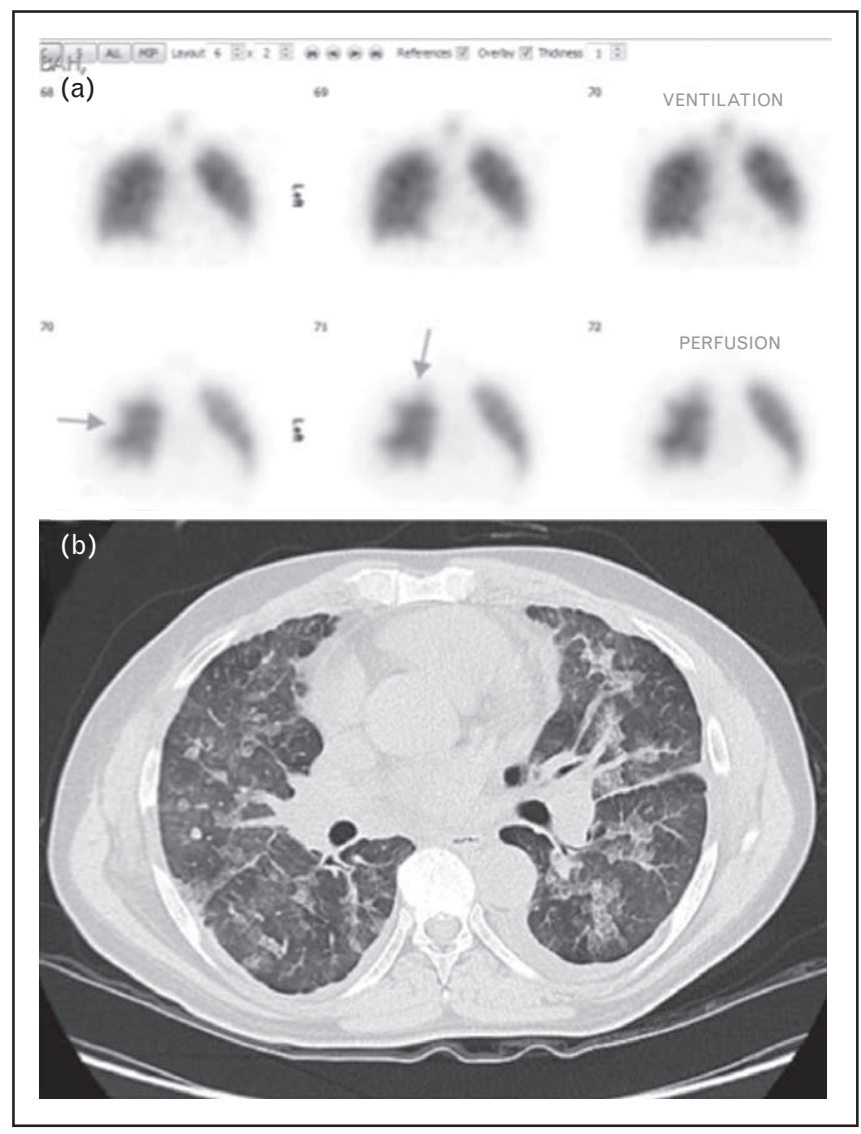

FIGURE 3. Radiology in PTTM. (a) Ventilation perfusion demonstrating sub-segmental defects in lung perfusion in a patient with PTTM. CTPA did not demonstrate these peripheral lesions (not shown). (b) High-resolution CT scanning in PTTM showing widespread ground glass opacification, faint nodules, thickening of the interlobular septa and small bilateral pleural effusions. CT, computed tomography; PTTM, pulmonary tumour thrombotic microangiopathy.

vascular limitation, with relative preservation of lung volumes but low gas transfer values.

Radionucleotide ventilation perfusion scanning usually shows multiple small peripheral sub-segmental perfusion defects not evident on CTPA, with normal ventilation (Fig. 3a). Serial ventilation perfusion may show resolution of perfusion defects following treatment $\left[15^{\prime \prime}\right.$.

The confirmation of pulmonary hypertension, once suspected clinically and suggested by a raised BNP with echocardiographic findings, is by right heart catheterization (RHC) ideally with pulmonary wedge aspiration cytology (see below). Precapillary pulmonary hypertension is defined by mPAP less than $25 \mathrm{mmHg}$ and PCWP less than $15 \mathrm{mmHg}$ [31]. The assessment of haemodynamic severity would be consistent with all other causes of pulmonary hypertension with signs of right heart dysfunction and low cardiac index being key prognostic factors [31].

\section{Radiological findings in pulmonary tumour thrombotic microangiopathy}

Plain chest radiography may be normal or show diffuse reticulonodular opacities or, less often, Kerley B lines and pleural effusions [32"]. Parenchymal computed tomography (CT) abnormalities (Fig. 3b) are nonspecific and include centrilobular nodules, ground glass attenuation, interlobular septal thickening and consolidation [8].

CT signs of pulmonary hypertension may be present including enlargement of the central pulmonary artery, right heart chamber enlargement and flattening of the intraventricular septum $\left[18,32^{\prime \prime}\right]$. Other pulmonary manifestations of malignancy may be present such as discrete metastatic deposits.

\section{Main HRCT findings}

Compared with pulmonary tumour embolism, where HRCT appearances are more often unremarkable, HRCT may show centrilobular nodularity, ground glass opacities (GGOs) and interlobular septal thickening (Fig. 3).

Centrilobular nodularity (i.e. small nodules in the centre of secondary pulmonary lobules) is often present in PTTM $[18,28,29]$. The nodularity is usually of an ultrafine granular appearance, and is likely to represent the peripheral pulmonary arterial lesions. These opacities are usually well defined as they reflect abnormalities in peripheral blood vessels; in bronchiolar disorders such as respiratory bronchiolitis, the nodule border is usually more blurred. Centrilobular nodularity on HRCT is an early sign in PTTM, and may disappear following chemotherapy [28], in keeping with resolution of the small vessel lesions. Quite frequently, the centrilobular nodules exhibit a tree in bud nodular pattern, a feature usually associated with plugging of respiratory bronchioles rather than vascular disease [33].

GGOs are reported in PTTM (often patchy or wedged shaped) $[18,29,34]$, for which the radiological differential diagnosis remains broad. In the setting of PTTM and pulmonary hypertension, especially with postcapillary involvement, GGO may be consistent with interstitial and airspace oedema [18], or interstitial inflammation, as suggested by resolution 1 month following highdose steroids [17"].

Interlobular septal thickening on HRCT (the corollary to septal lines on chest radiography) is 
described in PTTM, and is again a nonspecific finding. It simply indicates disease of the connective tissue, lymphatics and/or pulmonary veins. The nature and distribution of septal thickening may AQ7 provide clues to causes. In PTTM, it appears smooth and peripheral in distribution $[18,29,35]$, usually without pleural effusions. Lymphangitis carcinomatosa tends to cause more irregular, nodular interlobular septal thickening, resulting in prominence of the secondary pulmonary nodules, as well as thickening of the bronchovascular interstitium, subpleural nodules, pleural effusions, and often hilar or mediastinal node enlargement. Distinguishing PTTM from lymphangitis carcinomatosa may be difficult and the two conditions may coexist.

\section{Further assessment for malignancy}

Investigations in a patient with new-onset pulmonary hypertension and suspected PTTM should include a screen for undiagnosed malignancy. This may involve a full clinical assessment similar to the assessment of unprovoked PE [36]: mammography, possibly CT abdomen/pelvis and consideration of further targeted tests [15"]. This has formed part of our usual practice given the increased awareness of PTTM, although it is not specified in current pulmonary hypertension guidelines [31]. Tumour markers guide chemotherapeutic response [28] rather than diagnosis.

\section{Pulmonary wedge aspiration cytology and lung biopsy}

The diagnosis of PTTM can be made by the cytological examination of the aspirate from a wedged pulmonary artery catheter. This technique has a reported sensitivity of $80-88 \%$ and a specificity of $82-94 \%[5,6]$. It is reported in both PTTM and pulmonary tumour embolism [37], but would not be able to distinguish between these. Of note, if the sample is not wedged, malignant cells from 'upstream' (i.e. the liver) may contaminate the sample [7]. The sample should be heparinized, centrifuged and the buffy coat examined for malignant cells. Lung biopsy has been used to diagnose PTTM ante-mortem, either guided by CT, bronchoscopically or using video-assisted thoracoscopic surgery (VATS) $[26,28,34]$. However, we would advise caution in the presence of pulmonary hypertension.

\section{Positron emission tomography IN pulmonary tumour thrombotic microangiopathy}

The use of 2-(F-18)-fluoro-2-deoxy-D-glucose positron emission tomography (FDG-PET) has been reported in PTTM $[8,25,35]$, and for example showed uptake in areas of primary lung cancer and consolidation pathologically proven to be PTTM [8]. PTTM may be PET negative however [28], which may relate to size of lesions or that certain histological subtypes of gastric carcinoma are less FDG-PET-avid [38].

\section{MANAGEMENT}

Until recently, all case reports reported death soon after diagnosis. Anecdotal reports of therapeutic thrombolysis, unsurprisingly, have shown no benefit [39]. Ante-mortem diagnosis has enabled more aggressive treatment of the underlying cancer (using chemotherapy) and, potentially, the PTTM (using antiproliferative approaches). In some cases, a combined approach has been used for periods up to 14 months, alongside basic therapies including anticoagulation and oxygen therapy [34]. The role for conventional pulmonary vasodilators is uncertain. Current evidence is based purely on reported cases.

\section{Conventional pulmonary vasodilators}

Although no evidence suggests that the usual vasoactive mediators implicated in PAH are antiremodelling targets in PTTM, there is rationale for their use in abating pulmonary vasoconstriction. Endothelin receptor antagonists including bosentan [29] and ambrisentan $\left[15^{*}, 18\right]$ have been used. In some cases, PTTM progressed despite their use $\left[17^{*}, 18,29\right]$. In other cases, PTTM improved when pulmonary vasodilators were used in combination with imatinib $\left[15^{\prime \prime}, 29\right]$.

\section{Chemotherapy}

The reduction in number of malignant cells by chemotherapy might lessen the stimulus to fibrointimal proliferation. Reports suggest that early use is effective in that it may resolve cough and centrilobular nodules on HRCT [28]. A combination approach for example with dexamethasone, warfarin and aspirin [26] may be more beneficial, with therapeutic response seen with falling D-dimer, serum VEGF and tumour markers [28,34,40]. Efficacy is likely to depend on chemosensitivity [41].

\section{Anti-inflammatory/antiproliferative approaches}

These include broad 'anti-inflammatory' inhibition using glucocorticoids or specific blockade of relevant growth factors. The use of dexamethasone 
$(0.05 \mathrm{mg} / \mathrm{kg}$ daily, in combination with anticoagulation and chemotherapy) resulted in permanent resolution in one case of gastric cancer-associated PTTM [26], and to very short-term improvement in HRCT changes, cough, hypoxia and haemodynamics in a patient with recurrent breast carcinomaassociated PTTM [17"].

The tyrosine kinase inhibitor, imatinib, blocks phosphorylation of the PDGF receptor, and inhibits downstream cell growth. It is approved as an anticancer drug. Immunohistochemical studies support a basis for blockade of PDGF in PTTM $[4,8,9]$. PDGF signalling is also implicated in PAH [42], although clinical studies of imatinib in PAH have been complicated by adverse events including subdural haematomas in patients receiving anticoagulation [43]. Several reports suggest that imatinib may extend survival in PTTM $\left[1^{\prime}, 29\right]$, whereas imatinib led to reduced serum PDGF-BB and BNP levels alongside perfusion defects on ventilation perfusion, with persistent normalization of invasive pulmonary haemodynamics. Although pulmonary hypertension did not recur, both patients died of nonpulmonary hypertension systemic metastatic complications at 1012 months $\left[1^{\prime}, 29\right]$. Further to PDGF inhibition, VEGF inhibition using bevacizumab alone then in combination with imatinib was effective in treating pulmonary hypertension with an associated fall in serum VEGF levels. Again, the patient died of a nonpulmonary hypertension cause at 12 months [44]. These cases targeting specific growth factors are encouraging and require systematic assessment.

\section{Cancer and cancer treatment-related pulmonary hypertension}

Several treatment-related mechanisms may promote the development of pulmonary hypertension in patients with cancer. Chemotherapeutic agents including gemcitabine [45] and mitomycin-C may cause pulmonary veno-occlusive disease $[46,47]$, and the PDGF inhibitor dasatinib may actually cause PAH [48]. In addition, chemotherapy and radiation-induced left heart dysfunction may cause group 2 pulmonary hypertension [49]. Finally, cancer is an important risk factor for CTEPH [50].

\section{CONCLUSION}

PTTM should be considered in the differential diagnosis of a patient presenting with pulmonary hypertension of unknown origin, especially if they have a known underlying carcinoma. Autopsy studies indicate that PTTM is not exceedingly rare, and is likely to be underdiagnosed. Diagnosis can be difficult ante-mortem and depends upon a high index of suspicion when the clinical features are suggestive. Although the prognosis remains very poor, temporary responses to chemotherapeutic agents and pulmonary vasodilators may improve patient symptoms. Further understanding of this malignant cause of pulmonary hypertension is clearly needed.

\section{Acknowledgements}

The authors would like to thank Dr Angeles Maria Monteres, Consultant Pathologist, Royal Brompton Hospital, for her help with histological analysis.

The authors would also like to thank Dr Simon Padley, consultant radiologist, Royal Brompton Hospital, for his assistance reviewing radiological aspects.

\section{Financial support and sponsorship}

None.

\section{Conflicts of interest}

L.C.P. and S.J.W. have received honoraria and research funding from Bayer, GSK and Actelion Pharmaceuticals. A.U.C. has no conflicts of interest.

\section{REFERENCES AND RECOMMENDED \\ READING}

Papers of particular interest, published within the annual period of review, have been highlighted as:

- of special interest

-1. of outstanding interest

1. von Herbay A, Illes A, Waldherr R, Otto HF. Pulmonary tumor thrombotic microangiopathy with pulmonary hypertension Cancer 1990; 66:587-592.

2. Hughes JE. A case of hydatidiform mole with multiple small syncytial infarctions of the lungs. Proc R Soc Med 1930; 23:1157-1159.

3. Pinckard JK, Wick MR. Tumor-related thrombotic pulmonary microangiopathy: review of pathologic findings and pathophysiologic mechanisms. Ann Diagn Pathol 2000; 4:154-157.

4. Chinen K, Tokuda Y, Fujiwara M, Fujioka Y. Pulmonary tumor thrombotic microangiopathy in patients with gastric carcinoma: an analysis of 6 autopsy cases and review of the literature. Pathol Res Pract 2010; 206:682-689.

5. von Herbay A, Maiwald M, Ditton HJ, Otto HF. Histology of intestinal Whipple's disease revisited. A study of 48 patients. Virchows Arch 1996 429:335-343.

6. Kane RD, Hawkins HK, Miller JA, Noce PS. Microscopic pulmonary tumor emboli associated with dyspnea. Cancer 1975; 36:1473-1482.

7. Roberts KE, Hamele-Bena D, Saqi A, et al. Pulmonary tumor embolism: a review of the literature. Am J Med 2003; 115:228-232.

8. Uruga $\mathrm{H}$, Fujii $\mathrm{T}$, Kurosaki $\mathrm{A}$, et al. Pulmonary tumor thrombotic microangiopathy: a clinical analysis of 30 autopsy cases. Intern Med 2013; 52:13171323.

9. Okubo $\mathrm{Y}$, Wakayama $\mathrm{M}$, Kitahara $\mathrm{K}$, et al. Pulmonary tumor thrombotic microangiopathy induced by gastric carcinoma: morphometric and immunohistochemical analysis of six autopsy cases Diagn Pathol 2011; 6:27.

10. Uga $S$, Ikeda $S$, Matsukage $S$, Hamada M. An autopsy case of acute co pulmonale and paradoxical systemic embolism due to tumour cell microemboli in a patient with breast cancer BMJ Case Rep 2012; 2012:.

11. Buser M, Felizeter-Kessler M, Lenggenhager D, Maeder MT. Rapidly progressive pulmonary hypertension in a patient with pulmonary tumor thrombotic microangiopathy. Am J Respir Crit Care Med 2015; 191:711-712.

12. Gru AA, Pai RK, Roma AA. Pulmonary tumor thrombotic microangiopathy in patients with low-grade ovarian serous neoplasm: a clinicopathologic review of 2 cases of a previously unknown association. Int J Gynecol Pathol 2012; $31: 438-442$.

13. Tanaka $K$, Nakasya $A$, Miyazaki $M$, et al. A case of hepatocellular carcinoma with respiratory failure caused by widespread tumor microemboli. Fukuoka Igaku Zasshi 2011; 102:298-302.

14. Malani AK, Gupta C, Kutty AV, Betlej T. Pulmonary tumor thrombotic microangiopathy from metastatic gallbladder carcinoma: an unusual cause of severe pulmonary hypertension. Dig Dis Sci 2007; 52:555-557. 


\section{Disorders of the pulmonary circulation}

15. Minatsuki S, Miura I, Yao A, et al. Platelet-derived growth factor receptor-

- tyrosine kinase inhibitor, imatinib, is effective for treating pulmonary hypertension induced by pulmonary tumor thrombotic microangiopathy. Int Heart J 2015; 56:245-248

Recent important report of PDGF inhibition

16. Takahashi F, Kumasaka $T$, Nagaoka $T$, et al. Osteopontin expression in pulmonary tumor thrombotic microangiopathy caused by gastric carcinoma. Pathol Int 2009; 59:752-756.

17. Higashi A, Dohi $\mathrm{Y}$, Uraoka $\mathrm{N}$, et al. The potential role of inflammation

- associated with interaction between osteopontin and CD44 in a case of pulmonary tumor thrombotic microangiopathy caused by breast cancer. Intern Med 2015; 54:2877-2880.

Recent important report of role of inflammation.

18. Kumar N, Price LC, Montero MA, et al. Pulmonary tumour thrombotic microangiopathy: unclassifiable pulmonary hypertension? Eur Respir J 2015; 46:1214-1217.

19. Khan SA, Cook AC, Kappil M, et al. Enhanced cell surface CD44 variant (v6, v9) expression by osteopontin in breast cancer epithelial cells facilitates tumor cell migration: novel post-transcriptional, post-translational regulation. Clin Exp Metastasis 2005; 22:663-673.

20. Hashimoto-Kataoka T, Hosen N, Sonobe T, et al. Interleukin-6/interleukin-21 signaling axis is critical in the pathogenesis of pulmonary arterial hypertension. Proc Natl Acad Sci U S A 2015; 112:E2677-E2686.

21. Dorfmuller $P$, Gunther $S$, Ghigna MR, et al. Microvascular disease in chronic thromboembolic pulmonary hypertension: a role for pulmonary veins and systemic vasculature. Eur Respir J 2014; 44:1275-1288.

22. Caine GJ, Stonelake PS, Lip GY, Kehoe ST. The hypercoagulable state of malignancy: pathogenesis and current debate. Neoplasia 2002; 4:465-473.

23. Wahrenbrock $M$, Borsig $L$, Le $D$, et al. Selectin-mucin interactions as a probable molecular explanation for the association of Trousseau syndrome with mucinous adenocarcinomas. J Clin Invest 2003; 112:853-862.

24. Gainza E, Fernandez $S$, Martinez $D$, et al. Pulmonary tumor thrombotic microangiopathy: report of 3 cases and review of the literature. Medicine 2014; 93:359-363.

25. Ho AL, Szulakowski $P$, Mohamid WH. The diagnostic challenge of pulmonary tumour thrombotic microangiopathy as a presentation for metastatic gastric cancer: a case report and review of the literature. BMC Cancer 2015; 15:450.

26. Miyano S, Izumi S, Takeda $Y$, et al. Pulmonary tumor thrombotic microangiopathy. J Clin Oncol 2007; 25:597-599.

27. Simonneau G, Galie N, Rubin LJ, et al. Clinical classification of pulmonary hypertension. J Am Coll Cardiol 2004; 43 (suppl S):5S-12S.

28. Kayatani $\mathrm{H}$, Matsuo $\mathrm{K}$, Ueda $\mathrm{Y}$, et al. Pulmonary tumor thrombotic microangiopathy diagnosed antemortem and treated with combination chemotherapy. Intern Med 2012; 51:2767-2770.

29. Ogawa A, Yamadori I, Matsubara O, Matsubara H. Pulmonary tumor thrombotic microangiopathy with circulatory failure treated with imatinib. Intern Med 2013; 52:1927-1930.

30. Veinot JP, Ford SE, Price RG. Subacute cor pulmonale due to tumor embolization. Arch Pathol Lab Med 1992; 116:131-134.

31. Galie N, Humbert M, Vachiery JL, et al. 2015 ESC/ERS Guidelines for the diagnosis and treatment of pulmonary hypertension: The Joint Task Force for the Diagnosis and Treatment of Pulmonary Hypertension of the European Society of Cardiology (ESC) and the European Respiratory Society (ERS): Endorsed by: Association for European Paediatric and Congenital Cardiology (AEPC), International Society for Heart and Lung Transplantation (ISHLT). Eur Respir J 2015; 46:903-975.
32. Godbole R, Ghatol A, Betancourt J, et al. Pulmonary tumor thrombotic microangiopathy: clinical, radiologic, and histologic correlation. J Clin Imaging Sci $2015 ; 5: 44$.

Nice summary of clinico-patho-radiological correlation in PTTM.

33. Franquet $\mathrm{T}$, Gimenez $A$, Prats $\mathrm{R}$, et al. Thrombotic microangiopathy of pulmonary tumors: a vascular cause of tree-in-bud pattern on CT. AJR Am J Roentgenol 2002; 179:897-899.

34. Kitamura A, Nishimura N, Jinta $\mathrm{T}$, et al. A case of pulmonary tumor thrombotic microangiopathy diagnosed by transbronchial lung biopsy and treated with chemotherapy and long-term oxygen and anticoagulation therapies. Case Rep Pulmonol 2013; 2013:259080.

35. Tashima $Y$, Abe $\mathrm{K}$, Matsuo $\mathrm{Y}$, et al. Pulmonary tumor thrombotic microangiopathy: FDG-PET/CT findings. Clin Nucl Med 2009; 34:175-177.

36. Carrier M, Lazo-Langner A, Shivakumar S, et al. Screening for occult cancer in unprovoked venous thromboembolism. N Engl J Med 2015 373:697-704.

37. Bhuvaneswaran JS, Venkitachalam CG, Sandhyamani S. Pulmonary wedge aspiration cytology in the diagnosis of recurrent tumour embolism causing pulmonary arterial hypertension. Int J Cardiol 1993; 39:209-212.

38. Stahl $A$, Ott $K$, Weber WA, et al. FDG PET imaging of locally advanced gastric carcinomas: correlation with endoscopic and histopathological findings. Eur J Nucl Med Mol Imaging 2003; 30:288-295.

39. Keenan NG, Nicholson AG, Oldershaw PJ. Fatal acute pulmonary hypertension caused by pulmonary tumour thrombotic microangiopathy. Int J Cardiol 2008; 124:e11-e13.

40. Simonneau G, Robbins IM, Beghetti M, et al. Updated clinical classification of pulmonary hypertension. J Am Coll Cardiol 2009; 54 (Suppl):S43-S54.

41. Savage $P$, Roddie $M$, Seckl MJ. A 28-year-old woman with a pulmonary embolus. Lancet 1998; 352:30.

42. Ghofrani HA, Morrell NW, Hoeper MM, et al. Imatinib in pulmonary arteria hypertension patients with inadequate response to established therapy. Am J Respir Crit Care Med 2010; 182:1171-1177.

43. Hoeper MM, Barst RJ, Bourge RC, et al. Imatinib mesylate as add-on therapy for pulmonary arterial hypertension: results of the randomized IMPRES study. Circulation 2013; 127:1128-1138.

44. Higo $K$, Kubota $K$, Takeda $A$, et al. Successful antemortem diagnosis and treatment of pulmonary tumor thrombotic microangiopathy. Intern Med 2014 53:2595-2599.

45. Turco C, Jary M, Kim S, et al. Gemcitabine-induced pulmonary toxicity: a case report of pulmonary veno-occlusive disease. Clin Med Insights Oncol 2015; 9:75-79.

46. Perros F, Gunther S, Ranchoux B, et al. Mitomycin-induced pulmonary venoocclusive disease: evidence from human disease and animal models. Circulation 2015; 132:834-847.

47. Gagnadoux F, Capron F, Lebeau B. Pulmonary veno-occlusive disease afte neoadjuvant mitomycin chemotherapy and surgery for lung carcinoma. Lung Cancer 2002; 36:213-215.

48. Montani $\mathrm{D}$, Bergot $\mathrm{E}$, Gunther $\mathrm{S}$, et al. Pulmonary arterial hypertension in patients treated by dasatinib. Circulation 2012; 125:2128-2137.

49. Lipshultz SE, Cochran TR, Franco VI, Miller TL. Treatment-related cardiotoxicity in survivors of childhood cancer. Nat Rev Clin Oncol 2013; 10:697-710.

50. Kim NH, Lang IM. Risk factors for chronic thromboembolic pulmonary hypertension. Eur Respir Rev 2012; 21:27-31. 


\section{MCP}

Manuscript No. 220514

\section{Current Opinion in Pulmonary Medicine \\ Typeset by Thomson Digital \\ for Wolters Kluwer}

Dear Author,

During the preparation of your manuscript for typesetting, some queries have arisen. These are listed below. Please check your typeset proof carefully and mark any corrections in the margin as neatly as possible or compile them as a separate list. This form should then be returned with your marked proof/list of corrections to the Production Editor.

\section{QUERIES: to be answered by AUTHOR/EDITOR?}

\begin{tabular}{|c|c|c|}
\hline QUERY NO. & QUERY DETAILS & RESPONSE \\
\hline$<\mathrm{AQ} 1>$ & $\begin{array}{l}\text { As per style, the short title/running head } \\
\text { can have a maximum of } 65 \text { characters } \\
\text { including spaces and author names, and } \\
\text { abbreviations/acronyms only as } \\
\text { exceptions. Please check the suggested } \\
\text { short title, "Pulmonary tumour } \\
\text { thrombotic microangiopathy Price et } \\
\text { al." for appropriateness. }\end{array}$ & \\
\hline$<\mathrm{AQ} 2>$ & $\begin{array}{l}\text { Please confirm whether surnames/family } \\
\text { names (red) have been identified } \\
\text { correctly in the author byline. }\end{array}$ & \\
\hline$<\mathrm{AQ} 3>$ & $\begin{array}{l}\text { Please check the affiliations for } \\
\text { correctness. }\end{array}$ & \\
\hline$<\mathrm{AQ} 4>$ & $\begin{array}{l}\text { Please check correspondences details for } \\
\text { appropriateness. Also please note that } \\
\text { the correspondence details is identical } \\
\text { with the affiliation. }\end{array}$ & \\
\hline$<\mathrm{AQ} 5>$ & $\begin{array}{l}\text { For general style and clarity, language of } \\
\text { the article has been slightly modified at } \\
\text { certain places. Please check. }\end{array}$ & \\
\hline$<$ AQ6> & $\begin{array}{l}\text { Please define acronyms HRCT, PAH, } \\
\text { CTEPH, PASMC, ET-1, ESC, CTPA, } \\
\text { BNP, mPAP, PCWP, PE, PDGF-BB, } \\
\text { EVG, PDGFR, OPN, if possible. }\end{array}$ & \\
\hline$<\mathrm{AQ} 7>$ & $\begin{array}{l}\text { As per style, "aetiology" has been } \\
\text { changed to "causes". Please check. }\end{array}$ & \\
\hline$<\mathrm{AQ} 8>$ & $\begin{array}{l}\text { As the following references are outside } \\
\text { the review period, bullets and } \\
\text { annotations have been deleted as per } \\
\text { style. Refs. }[1,29] \text {. }\end{array}$ & \\
\hline
\end{tabular}


$\mid<$ AQ9 $>\quad$ Please check whether the single page detail provided in Refs. [9, 25, 32, 34, $41]$ are correct. If not, please provide the page range detail.

$<$ AQ10 $>\quad$ Please provide page range for Ref. [10].

$<$ AQ11 $\quad$ As per style, legends to figures should be as short as possible and information given in the legend should not duplicate that given in the text. Comments on the figures must appear in the text only.

Please consider rewording the legend to Fig. 2 in view of these requirements. 